\title{
University/industry cooperative teaching laboratory for undergraduate education
}

\section{James Leger}

James R. Leger, "University/industry cooperative teaching laboratory for undergraduate education," Proc. SPIE 4588, Seventh International Conference on Education and Training in Optics and Photonics, (28 May 2002); doi: $10.1117 / 12.468722$

SPIE Event: Education and Training in Optics and Photonics 2001, 2001, Singapore, Singapore 
Invited Paper

\title{
University/Industry Cooperative Teaching Laboratory for Undergraduate Education
}

\author{
James R. Leger \\ Department of Electrical and Computer Engineering \\ University of Minnesota \\ 4-174 EE/Csci Building \\ 200 Union Street SE \\ Minneapolis MN, 55455
}

\begin{abstract}
An undergraduate optics laboratory sponsored by the National Science Foundation of the United States was established to foster a link between local industry and academia. A series of innovative experiments was developed utilizing highspeed data acquisition equipment and signal processing software to demonstrate the fundamentals of diffraction, fiber optics, and physical optics principles. The experiments were performed in two complementary settings. The university experiments concentrated on basic optical principles and experimental techniques. A parallel industrial component was provided by local industry. Students were invited to industrial research laboratories to work on real-life optical problems of current interest. The students were able to see the relevance between fundamental optical principles and real industrial problems, use state-of-the-art equipment, and experience working in an industrial laboratory. Feedback was also solicited from industry management regarding improvements to academic training of students for the work force.
\end{abstract}

\section{Introduction and Course Rationale}

University coursework and teaching laboratories often emphasize a formal approach to learning, where fundamentals are stressed, basic tools are developed, and laboratory exercises demonstrate basic physical phenomena. In contrast, the work of a practicing engineer in industry is very often interdisciplinary, may be tempered by practical concerns such as cost and practicality, and often utilizes specialized experimental equipment. The purpose of this undergraduate laboratory is to blend these two learning experiences together into a single course. The university portion of the laboratory uses state-of-the-art measurement equipment and signal processing software to demonstrate fundamental concepts. The industrial component is designed to give the students first-hand experience on problems that are of current interest to industry. The industrial component was structured to emphasize 1) relevance to company products, 2) utilization of specialized equipment, and 3) data analysis and documentation procedures.

The goals of the university laboratory component are:

- Increase awareness of undergraduates to optics

- Introduce fundamental optical principles and techniques

- Introduce techniques of modern instrumentation

- Practice proper experimental documentation skills

Documentation skills are emphasized by requiring each student to maintain a laboratory notebook. The documentation contained in this notebook is expected to meet the legal standards required to protect intellectual property in an industrial setting. Thus, each notebook page is dated, signed, and witnessed. The descriptions of procedures and references to previous work are expected to be clear and complete. All data is expected to be analyzed thoroughly and important conclusions drawn. All entries are recorded in pen and all changes initialed and dated. 
The goals of the industrial laboratory component are:

- Introduce students to industrial laboratory practices

- Give students an opportunity to work on real-world problems

- Provide access to specialized state-of-the-are equipment

- Improve the dialog between industry and the university

The course at the University of Minnesota is designed so that undergraduates will have the proper prerequisites by their senior year. Core courses that are required for understanding the laboratory material include:

- Two-year calculus curriculum including multi-variable calculus, partial differential equations and linear algebra

- Electromagnetic fields and waves

- Semiconductor devices

- Signals and systems

- Statistical methods

In addition, the students are asked to complete a companion course in Physical Optics, or to take this course concurrently with the laboratory. Although the University of Minnesota also offers undergraduate courses in Optical Fiber Communication, Optical Electronics and Lasers, and Optical System Design, these courses are not required for the current configuration of the laboratory.

Even though the course is designed to be accessible to undergraduates, at least half of the enrollment has come from first and second-year graduate students. The core prerequisites are waived for these students. However, concurrent enrollment in the Physical Optics course is still required. We have found that both Physics and Electrical Engineering students are very comfortable with the material in the course. Students from Mechanical Engineering and Chemical Engineering also have taken the course and performed well. However, extra preparation is sometimes required to insure a sufficiently deep level of understanding of signals and systems.

\section{University Laboratory Equipment}

One of the goals of this project was to make data acquisition and analysis as painless as possible. We have designed most of the laboratory experiments around a CCD camera interfaced to a personal computer through a frame grabber. We are currently using the laser beam analyzer hardware manufactured by Spiricon, Inc. This allows us to capture an entire two-dimensional frame of data very easily, as well as perform simple data manipulations such as frame averaging, pseudo-color and three-dimensional plotting, Gaussian fitting, etc. These data are passed to the PC, where data analysis software such as MatLab can readily be accessed for more sophisticated data processing and graphics. We have also found it useful to have a conventional television monitor for direct display of the CCD image, as well as a thermal printer for providing hard copy of the television image. These pictures can be pasted directly into the students' notebooks to document their observations. A color computer plotter completes the light analysis hardware, and is useful for generating hard copies of pseudo-color pictures and computer-generated data.

Although CCD cameras simplify data collection, they also have their limitations. The background noise level of an uncooled CCD often makes it difficult to measure low light levels. We use extensive temporal averaging to reduce this time-varying noise. Even so, the dynamic range of the CCD is limited and many light fields such as diffraction patterns are hard to measure accurately. It is very important to supply some sort of neutral density filters to control the amount of light incident on the CCD. Standard fixed filters that fit over the CCD camera are used, but are found to be a bit cumbersome. We also provide a continuously variable neutral density wheel (usually placed directly after the laser) for convenient and accurate adjustment of light. When very large dynamic range imagery is present, we have found it necessary to take multiple images of the same field using different light attenuation settings. If there is sufficient overlap of the linear ranges between successive images, they can be calibrated with respect to each other and an extended 
dynamic range plot can be generated. Although this gives satisfactory qualitative results, the accuracy of such measurements is compromised by the assumed linearity of the CCD, and very large dynamic range measurements often contain significant experimental error.

For experiments requiring coherent light, we use $7 \mathrm{~mW}$ HeNe lasers. Standard spatial filtering and collimating optics complete this set-up. For experiments requiring incoherent light, standard high intensity halogen sources have proved to be sufficient. Experiments with spatial coherence often require bright incoherent sources such as arc lamps. However, we have found that the CCD-based sensors provide sufficient sensitivity so that good results can be achieved with simple lamps.

Finally, all labs are set up on an optical breadboard. In principle, the breadboards can be modified to provide vibration isolation. However, for most of our experiments, this is unnecessary. In one case (Fourier Transform spectrometer experiment), the fringe stability is compromised by table vibrations. However, by capturing data in a continuous stream and then analyzing it in a frame-by-frame manner, we were usually able to find frames where the vibration effect is minimized. Although this is not a substitute for true vibration isolation, it is a significant improvement over a direct measurement of a single frame.

\section{University Laboratory Exercises}

The laboratory course consists of nine exercises:

- Fundamentals

1. Basic techniques, interferometry, and aberrations

2. Fresnel diffraction

3. Fraunhofer diffraction

4. Temporal coherence and Fourier transform spectroscopy

5. Spatial coherence and speckle

- Applications

6. Imaging (MTF, aliasing, contrast inversion with misfocus, etc.)

7. Spatial filtering (Schlieren optics, edge enhancement, raster line removal, etc.)

8. Properties of single-mode and multi-mode fibers

- Industrial

9. Industrial project

In this section, we will briefly outline the content of the university exercises emphasizing fundamental principles and applications.

\subsection{Basic techniques, interferometry, and aberrations}

The first lab introduces students to basic laboratory technique. A plane parallel plate (uncoated glass etalon polished flat to $\lambda / 20$ on both sides) is used as a simple shearing interferometer. ${ }^{1}$ The interferometer is used to measure misfocus and spherical aberration quantitatively. Fig. 1 shows the results from measuring a laser beam with both these aberrations. The plane parallel plate is then used to adjust afocal optical systems, locate the front focal point of a lens with an auxiliary lens, and obtain a quantitative measure of spherical aberration from a simple lens. 

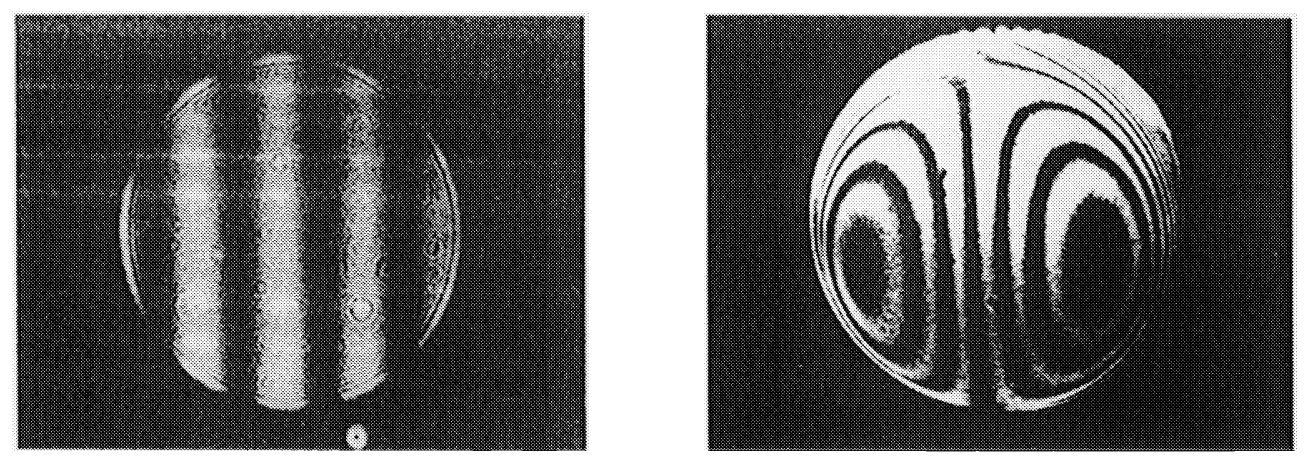

Fig. 1. Fringe patterns from shearing interferometer. a) misfocus, b) spherical aberration.

\subsection{Fresnel diffraction}

Fresnel diffraction is demonstrated by observing diffraction from simple objects. The simplest beam for observing Fresnel diffraction is the Gaussian beam from the HeNe laser. The Spiricon software is used to fit a Gaussian curve to the measured laser beam, and the beam size is measured as a function of propagation distance. These curves are fit to standard Gaussian beam propagation equations. An interesting extension can be added to this experiment for the more advanced student. The Gaussian beam can be slightly aberrated and a measurement of $\mathrm{M}^{2}$ conducted by calculating the second moments of the intensity function. ${ }^{2}$ The invariance properties of $\mathrm{M}^{2}$ can then be explored with this aberrated beam.

Fresnel diffraction from a circular aperture is also explored, and the students are asked to measure the propagation distances corresponding to different occurrences of the Poisson spot. This experiment is ideal for making the connection between a simple Fresnel zone construction and the more complete Fresnel integral. Finally, the students are asked to measure the intensity of the "dark" and "bright" Fresnel spots, and compare them to the theory based on the simple Fresnel integral.

By far, the most entertaining demonstration of Fresnel diffraction results from observing the Talbot effect. ${ }^{3}$ Simple Talbot theory predicts that a periodic object will form a self-image at a distance $Z_{\mathrm{T}}=2 \mathrm{~d}^{2} / \lambda$, due simply to Fresnel diffraction. In this case, $d$ is the period of the object and $\lambda$ is the wavelength of light. The effect is very easy for a student to understand if she has mastered the concept of the angular plane wave description of a light field. The experiment dramatically demonstrates this self-imaging effect. Although any periodic object could be used in principle, we use a simple two-dimensional grating with a $25 \%$ duty factor. The more advanced concept of the Fractional Talbot effect $^{4}$ can also be explored in this experiment. At certain fractional planes ${ }^{5}$, multiple images are formed. Experimental results from this experiment are shown in fig. 2.

\subsection{Fraunhofer diffraction}

Fraunhofer diffraction studies give the student an opportunity to explore the various aspects of the two-dimensional optical Fourier transform. The set-up for this lab is a simple Fourier processor, with the CCD camera in the back focal plane of a lens. The following simple experiments are performed:

1. Diffraction from simple apertures (square, circle, two circles). The intensity patterns are compared to theory in each case. The $1 / \mathrm{r}^{3}$ asymptotic decay from an Airy disk is compared with the asymptotic decay from a square aperture of $1 / \mathrm{r}^{2}$ along the side of the square and $1 / \mathrm{r}^{4}$ along the diagonal.

2. Double slits of varying spacing are used to demonstrate the scaling theorem of Fourier transforms. 


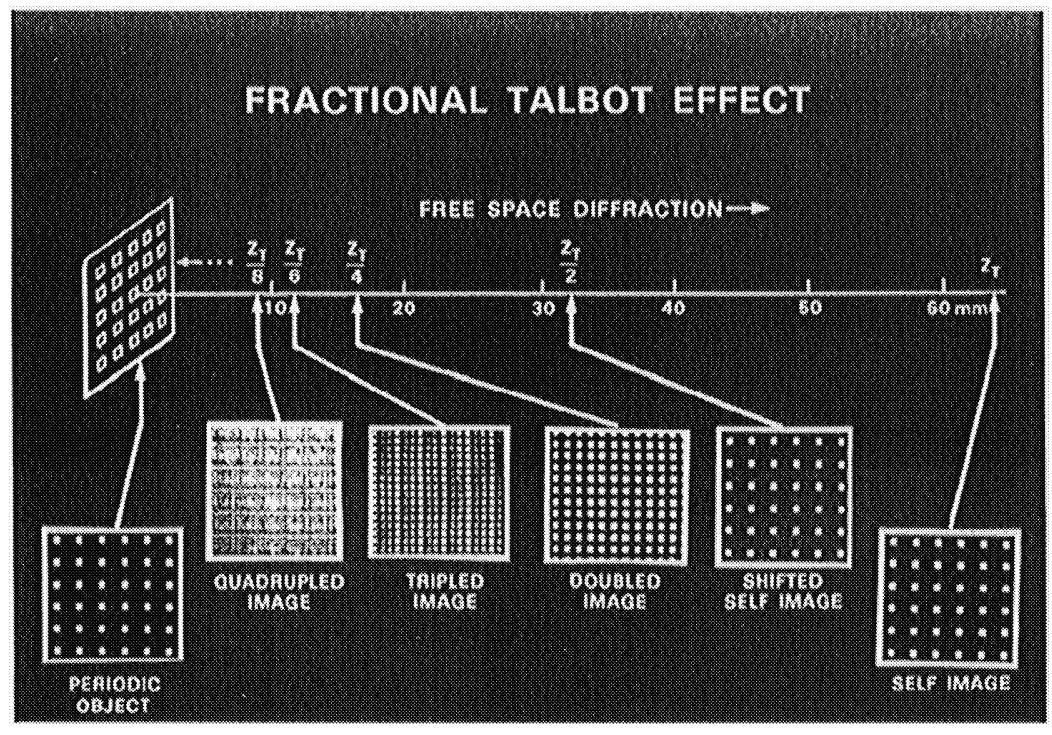

Fig. 2 Fresnel diffraction patterns illustrating fractional Talbot effect.

3. The resolution of a grating $\lambda / \Delta \lambda=\mathrm{Nq}$ is demonstrated, where $\lambda$ is the center wavelength, $\Delta \lambda$ is the wavelength resolution, $\mathrm{N}$ is the number of grating lines, and $\mathrm{q}$ is the diffraction order of the grating. Diffraction patterns from gratings composed of between one and six lines are observed and related to the grating resolution equation.

4. The translation invariance property of the Fourier transform is demonstrated by moving an object in the front focal plane and observing the intensity in the back focal plane of a lens.

5. The symmetry properties of a two-dimensional Fourier transform are explored using real objects (i.e. no phase component) with varying symmetries.

6. The convolution theorem is demonstrated by placing a simple aperture in contact with a grating. The diffraction pattern is seen to consist of a convolution between the two individual diffraction patterns.

A series of more advanced demonstrations is also included in this lab exercise:

1. Apodization experiments are difficult to perform because apodizing apertures are hard to fabricate, and the attenuated side-lobes are hard to measure. We introduce the concept of apodization by studying the diffraction effects from central obscurations. The effect is described as an "inverse apodization", since the side lobe intensities are seen to increase whereas the central lobe width decreases. Students familiar with digital signal processing immediately recognize the correspondence between optical apodization and windowing of an FFT.

2. The autocorrelation theorem and the Fourier transform of random processes are explored by comparing the diffraction patterns from a slide containing many randomly placed identical apertures with a slide containing randomly placed pairs of apertures. The appearance of a cosine modulation in the diffraction pattern of the second slide can be understood in several ways. We explain that one measure of the object spatial statistics is given by the autocorrelation of the object. The fact that many of the circles are paired up in the same way in the second slide generates peaks in the autocorrelation function at +- the aperture pairing distance. Since the 
intensity of the diffraction pattern is given by the Fourier transform of the autocorrelation function of the original slide, this correlated behavior manifests itself as a cosinusoidal modulation of the diffraction pattern.

3. The Mills Cross is an arrangement of circular apertures in the form of a "+" sign. The diffraction pattern from this can be intuitively explained, and gives students a chance to exercise their thinking in the Fourier domain.

4. Optical pattern recognition experiments are conducted by observing the diffraction patterns from slides taken of various handwriting samples. Several different writers were asked to copy two different paragraphs of text using cursive script. The diffraction patterns are then observed for common features of a single writer that are independent of the actual text. Results from this experiment are shown in fig. 3 for two of the writers. The students can also compare diffraction patterns from Chinese writing as well as printed text.

5. The optical Fourier transform can be compared with a digitally obtained Fourier transform by using an FFT. The data is input to the computer by imaging the slide onto the CCD and capturing the frame corresponding to the object data. A two-dimensional FFT is then performed and the result is compared with its optical counterpart.
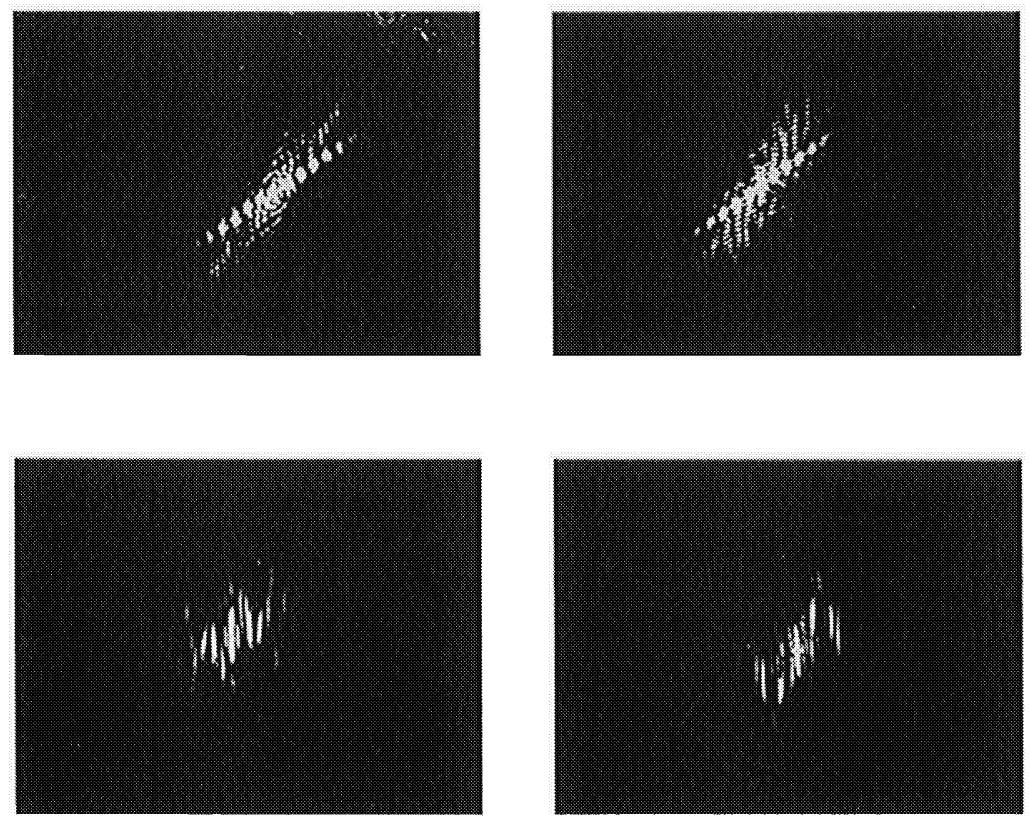

Fig. 3. Fraunhofer diffraction pattern from handwriting. a) Writer \# 1, first text. b) Writer \# 1, second text. c) Writer \#2, first text, d) Writer number 2, second text.

6. A periodic signal buried in noise provides an effective demonstration of "processor gain" afforded by a Fourier transform. A series of identical, equally spaced lines provides the signal pattern. The second pattern contains the same pattern as the first with many randomly spaced and oriented lines superimposed. This represents a periodic signal immersed in noise. Since the noise has an approximately flat Fourier spectrum, its diffraction 
pattern is spread out over a wide range of spatial frequencies. However, the periodic signal gives rise to spikes in the diffraction pattern, and is immediately recognizable even in the presence of significant noise.

\subsection{Temporal coherence and Fourier transform spectroscopy}

This experiment starts with the construction of a Michelson interferometer. The length of one interferometer arm is adjusted by moving the position of the mirror on a rail. A tilt is provided to one of the return mirrors so that the interferometer output consists of several fringes. The CCD camera is then used to measure the visibility of these fringes. As mentioned above, this setup is very susceptible to vibration. However, by taking multiple sets of data corresponding to many successive frames at each mirror position, the data can be scanned for ones with the best visibility (corresponding to the least vibration noise). We have found that the magnitude of the noise that corrupts our data tends to vary greatly in time, and is often virtually absent for short periods of time. This technique has allowed us to get reasonably noise-free data in a rather noisy environment.

Data is taken of the fringe pattern visibility for various differential arm lengths (producing the magnitude of the autocorrelation function). Since our HeNe laser supports several longitudinal modes, the visibility data show the distinct peaks and nulls corresponding to the longitudinal coherence properties of the laser. We are not able to recover the phase of the autocorrelation function due to the fringe drift in our set-up. Thus, it is not possible to actually invert the data and recover the spectrum using an FFT.

\subsection{Spatial coherence and speckle}

This experiment uses a halogen lamp projected through a small square aperture to study spatial coherence. The light from the incoherent aperture is passed through two slits and the interference pattern observed in the back plane of a lens. A red camera filter or a Wratten infrared filter is used to reduce the bandwidth of the light source and provide a more accurate fringe pattern. The fringe visibility is then measured as a function of double-slit spacing to generate a onedimensional spatial coherence function. The main lobe of the coherence function is then compared with theory.

More complex apertures are then used as incoherent sources and the Fourier relationship between the aperture intensity and the complex coherence function is verified (Van Cittert-Zernike theorem). We have found that two adjacent square apertures gives excellent results, and several of the lobes of the coherence function can be easily seen. It is also easy to measure the phase shift of the interference pattern and verify the signs of each lobe in the coherence function.

Students appreciate observing the interference pattern with their eyes (using a small bench microscope) while the source size and slit spacing are varied. In our set-up, the incoherent source is effectively imaged by the lens, so the effect of enlarging the source is clearly seen as blurring the interference pattern (and reducing the visibility function).

There are several other effects that can be demonstrated with this set-up. The light source can be imaged onto the pinhole with varying magnification, and the resultant intensity observed in the interference pattern. Since the final intensity is governed by the radiance of the source rather than the total power, one sees that the total power at the CCD is not affected by source magnification. This provides an effective demonstration of the radiance (or brightness) theorem.

Speckle statistics are demonstrated in this laboratory exercise by illuminating a diffuser (a piece of frosted tape) with the $\mathrm{HeNe}$ laser and imaging the result onto the CCD. Several effects are observed qualitatively, including the average speckle size as a function of lens aperture size, and the modulation depth of the speckle. Quantitative data are then obtained by recording an image of the speckle pattern when a specific rectangular pupil is used in the aperture plane of the imaging lens. A second speckle pattern is recorded using a mask containing two square holes as the pupil. Fig. 4 shows typical speckle patterns recorded with the single aperture (4a) and the double aperture (4b). The computer is then used to calculate histograms as well as the Fourier power spectra of the speckle patterns. The results are compared with 
the theory of speckle. Fig. 4c shows a typical result obtained from calculating the power spectrum of the speckle pattern from a two-hole aperture.

This experiment can be used to demonstrate several basic results from statistical optics. A connection can also be made between coherent imaging, spatially random phase imaging, and incoherent imaging. Finally, basic issues of power spectral estimation are encountered in this experiment. The student will find that a simple FFT of the data in fig. 4b leads to a very noisy estimate of the spectrum due to the statistical instability of the Fourier transform. Spectral averaging or other similar techniques are required to obtain a reasonably noise-free spectral estimate. The graph shown in fig. $4 \mathrm{c}$ was produced by calculating the squared magnitude of the Fourier transform of each line of data in fig. $4 \mathrm{~b}$, and averaging all these one-dimensional power spectra.

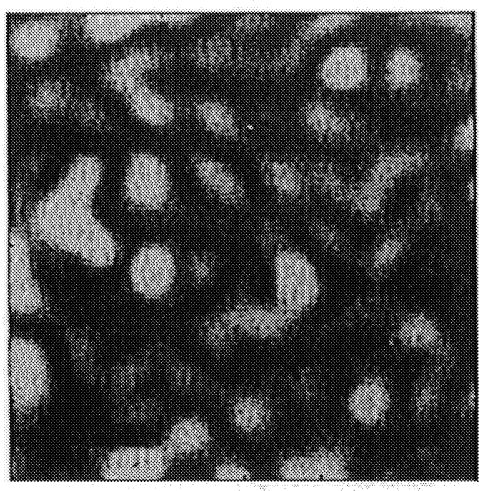

(a)

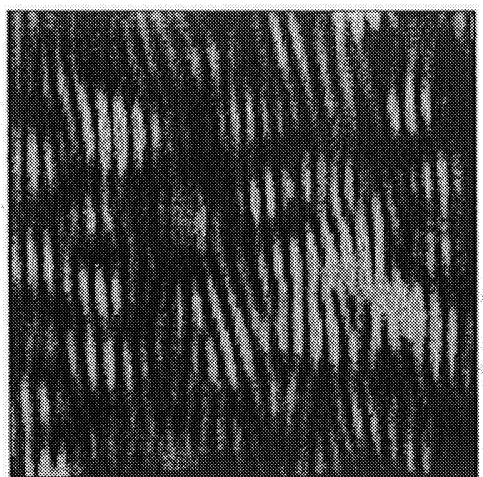

(b)

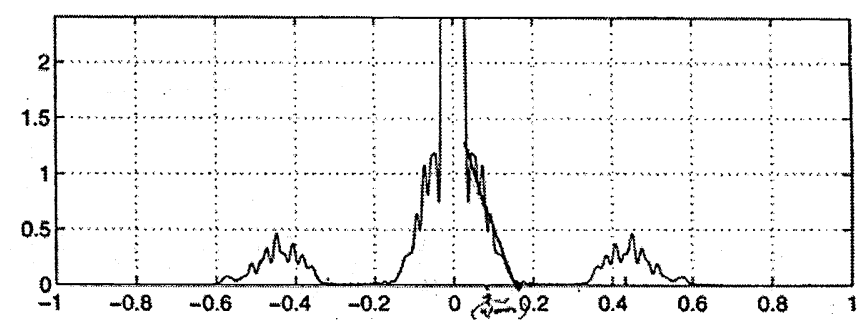

(c)

Fig. 4. Speckle statistics. a) Speckle pattern from single square pupil b) Speckle pattern from double square pupil. c) Average Fourier power spectrum of speckle in (b).

\subsection{Coherent and incoherent imaging}

Coherent imaging and spatial filtering is demonstrated with a traditional afocal imaging system. The transverse and longitudinal magnification properties are explored. Then, many of the classical spatial filtering experiments are performed. These include:

1. Low-pass filtering

2. High pass filtering and edge enhancement

3. Half-tone removal 
4. Raster line removal

5. Schlieren phase imaging

The half-tone and raster line removal experiments illustrate the concepts of aliases very visually, since the student is able to see the multiple diffraction orders (aliases) in the Fourier plane of the optical system and it is simple to understand that the sampling results from interference between these orders. Elimination of all but one order removes the sampling.

Schlieren optics are constructed simply by blocking half of the Fourier spectrum (either including or excluding the zero order). Although we have tried several phase objects, we have found that a hot soldering iron gives an impressive result and is very easy to produce.

Incoherent imaging is performed by placing a simple TV camera lens on the CCD and using this system to observe incoherently illuminated objects. We use a simple reproduction of a spoke target similar to that found in many Fourier optics books ${ }^{6}$. Using this object, we perform a qualitative and quantitative study of contrast reversal that occurs in misfocused optical systems. It is very easy to produce this contrast inversion by changing the degree of misfocus and the lens aperture. The student is asked to remark on the effect of varying these two parameters. The MTF of the focused and misfocused system is then crudely determined by using the computer imaging software. Different horizontal scans of the spoke target produce sine waves of varying frequency, and the visibility of these sine waves can be measured quantitatively. The contrast inversion can be easily seen to result in a 180 degree phase shift of the resulting sinusoidal signal.

A final simple experiment shows the students the effects of optical aliasing. Here, the CCD image is digitized by the computer and decimated by varying amounts to correspond to different sampling rates. The spoke target provides an object with known spatial frequencies, and the effects of aliasing are easily understood. Fig. 5 shows a dramatic example of higher spatial frequencies being represented as lower ones. The point where a spatial frequency aliases to the dc value is easily seen as the maximum extent of the flare in fig. 5. Close inspection shows regions where the higher-order harmonics of the binary spoke target alias to lower frequency values whereas the fundamental is still accurately represented at the proper frequency.

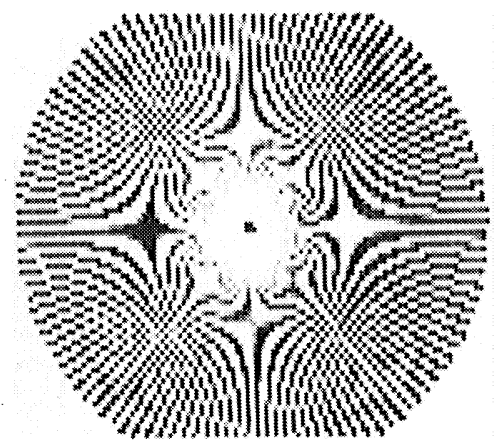

Fig. 5 Optical aliasing from a spoke target imaged with coarse resolution

\subsection{Single and multi-mode fiber properties}

Our experiments with fibers are limited to optical propagation effects. We perform the following experiments with a $\mathrm{HeNe}$ laser as a source:

1. Practice technique of cleaving and splicing single and multi-mode fibers. Measure coupling efficiency into both fiber types.

2. Measure the numerical aperture of a multimode fiber by viewing the far-field pattern 
3. Measure the bending loss of a multimode fiber as a function of bend angle.

4. Measure the output of a multimode fiber. Observe speckle effects, sensitivity to vibration and pressure, and polarization effects.

5. Excite various low-order modes in a $1300 \mathrm{~nm}$ single-mode fiber using a $\mathrm{HeNe}$ laser ( $633 \mathrm{~nm}$ wavelength).

6. Observe the mode structure of a $633 \mathrm{~nm}$ single-mode fiber.

7. Observe fiber birefringence and the effect of stress on birefringence.

All of these experiments employ the basic image analysis equipment of the previous labs.

\section{Industrial Laboratory Exercises}

We have worked with several different companies to offer the industrial component of this laboratory experience. The industrial lab exercises have generally fallen into three categories: 1) the lab involves fundamental measurements of an optical parameter using sophisticated equipment developed by the company, 2) the lab tests the performance of a specific company product, or 3 ) the lab is a (simplified) version of an actual exploratory experiment recently performed by the company as part of product development. An example of each of these lab types is described below.

\subsection{Measurement of diffractive optic MTF}

Honeywell Inc. has developed an expertise in design and fabrication of diffractive optics. As part of this effort, a special purpose instrument to measure lens MTF has been designed. The MTF measurement technique is based on recording the response to an illumination step function. The Fourier transform of the derivative of the edge response produces a high quality MTF measurement of both diffractive optical elements and conventional optics. The students were asked to measure several different types of lenses, observe the degradation in MTF from spherical aberration, and compare refractive and diffractive lens MTFs. This experiment complemented the university lab exercise on imaging, since lens MTFs were measured in both lab exercises but in completely different ways. The students were able to contrast the two different measurement methods, as well as have first-hand experience measuring state-of-the-art diffractive structures.

\subsection{Measurements of Fresnel lenses and specialty fibers}

The 3M Company fabricates many optical devices for commercial applications. One ubiquitous device is an inexpensive plastic Fresnel lenses used on overhead projectors, traffic lights, etc. In the first part of this laboratory experience, the students were asked to measure several properties of these lenses and compare them to conventional lenses. Total lens efficiency was measured as well as imaging resolution. The theory of Fresnel lenses was also presented to enhance the lab. In this case, the test equipment consisted of simple light sources and detectors. However, the students were able to experiment with a variety of Fresnel devices, as well as see how these devices were used in actual products.

The second half of the lab consisted of measuring the performance of several specialty fibers. Both polarization maintaining and polarizing fibers were available. In this case, commercial equipment for measuring index profiles, the $\mathrm{H}$-parameter, and specific fiber performance was employed. The complete fiber fabrication process, including MCVD growth and fiber drawing were also demonstrated. In both these lab experiences, the students were able to explore the optical properties of special devices fabricated in the research labs of the company.

\subsection{Experiments with temperature-sensitive phosphors}

Industrial sensors are one of Rosemont Inc.'s major product lines. As an exploratory effort to make a fiber optic temperature sensor, Rosemount engineers had designed a test bench to compare the dependence of florescence decay time on temperature for a wide variety of materials. The students were invited to participate in this experiment, taking data that was directly useful to the company research. This laboratory experience gave the students a chance to become directly involved in an industrial research project. 


\section{Conclusions}

A university/industry cooperative teaching laboratory has been described that emphasizes both fundamental optical principles and real-world experiences. Student reaction to the industrial component has been overwhelmingly positive, with many commenting that this has been their first chance to view an industrial research laboratory. We believe that this program has strengthened the ties between the University and local industry, and that both sides have benefited from the interaction.

\section{References}

1. D. Malacara, ed., "Optical shop testing," Wiley, New York (1978).

2. A.E. Siegman, in: Solid state lasers: new developments and applications, M. Inguscio and R. Wallenstein, eds., NATO Advanced Study Institute Series, Series B: Physics 317, 13-28 (1993).

3. W.H.F. Talbot, Philos. Mag. 9, 401-407 (1836).

4. J.T. Winthrop and C.R. Worthington, J. Opt. Soc. Am. 55, 373 (1965).

5. J.R. Leger and G.J. Swanson, Opt. Lett. 15, 288-290 (1990).

6. J. Goodman, "Introduction to Fourier Optics," McGraw-Hill, New York (1996).

\section{Acknowledgments}

The author would like to acknowledge a generous grant form the National Science Foundation (Grant \# NSF-DUE9451213) as well as support from the Institute of Technology at the University of Minnesota. Participation by the Honeywell Corporation, 3M, Rosemount, and CyberOptics is also recognized and appreciated. The industrial experiments described in this paper were developed by Allen Cox, Dawn Tilstra, and Madelene Flemming. The student work displayed was taken from the notebooks of Neophytos Morphis, Bruce Thomas, and Keesook Han. 